Shintaro Kikuchi • Akito Tanoue • Fumio Endo

Shoji Wakasugi • Nobutake Matsuo • Gozoh Tsujimoto

\title{
A novel nonsense mutation of the PEPD gene in a Japanese patient with prolidase deficiency
}

Received: November 8, 1999 / Accepted: November 24, 1999

\begin{abstract}
A nonsense mutation at amino acid residue 184 in the human peptidase $\mathrm{D}(P E P D)$ gene caused the production of a truncated polypeptide. Characterizing molecular defects in patients provides clues to elucidate the relationship between the phenotype and the genotype.
\end{abstract}

Key words PEPD $\cdot$ Prolidase deficiency $\cdot$ Mutation $\cdot$ Polymorphism $\cdot$ Nonsense mutation

\section{Introduction}

Prolidase deficiency is a rare genetic disease inherited as an autosomal recessive trait. It has a heterogeneous phenotype associated with chronic ulcerative dermatitis, mental retardation, and massive urinary excretion of imidodipeptides. The age at onset of the disease varies from birth to adult, and some cases are asymptomatic. The striking variation in the clinical phenotype associated with prolidase deficiency is largely unexplained.

The clinical features of the skin lesions are largely caused by the inhibition of normal recycling of proline, which causes an alteration in the metabolism of collagen and other proline-rich proteins (Isemura et al. 1981). It has also been suggested that prolidase plays a critical role in wound heal-

S. Kikuchi $\cdot$ A. Tanoue $\cdot$ G. Tsujimoto $(\square)$

Department of Molecular and Cellular Pharmacology, National

Children's Medical Research Center, 3-35-31 Taishido, Setagaya-ku,

Tokyo 154-8509, Japan

Tel. +81-3-3419-2476; Fax +81-3-3419-1252

e-mail: gtsujimoto@nch.go.jp

F. Endo

Department of Pediatrics, Kumamoto University School of

Medicine, Kumamoto, Japan

S. Wakasugi

Department of Dermatology, Kumamoto University School of

Medicine, Kumamoto, Japan

S. Kikuchi $\cdot$ N. Matsuo

Department of Pediatrics, Keio University School of Medicine,

Tokyo, Japan ing (Oono et al. 1997). The underlying pathogenesis for the other manifestations, such as mental retardation, is not known.

Several mutations in the PEPD gene have been reported. However, the relationship between phenotype and genotype is not clear. Patients with mental retardation lack expression of transcripts of the PEPD gene (Endo et al. 1990), but discrepancies in clinical symptoms between siblings with the same mutation have been pointed out (Isemura M. et al. 1979; Tanoue et al. 1991). This suggests that additional factors not related to the mutation may modify the expression of the disorder.

Here, we analyze a Japanese patient with severe symptoms of prolidase deficiency and describe a novel mutation associated with this deficiency.

\section{Patient and methods}

Patient

The patient is a member of a large family pedigree in which a brother and a cousin also suffer from prolidase deficiency (Ogata et al. 1981). They were characterized by recalcitrant leg ulcers that had been present since birth, characteristic faces with saddle nose, mental retardation, frequent infections, partial deafness, visual disturbances, and joint dislocations. Prolidase activity in red blood cells (RBCs) could not be detected, and cross-reacting materials (CRMs) were not detectable by Western blot analysis (Endo et al. 1990).

Cells

Skin fibroblasts were established from skin biopsies. Cultured skin fibroblasts were grown in Dulbecco's minimal essential medium containing $10 \%$ fetal calf serum (FCS) at $37^{\circ} \mathrm{C}$ in a $5 \% \mathrm{CO}_{2}$ atmosphere as described (Tanoue et al. 1990a). 
Isolation of DNA and RNA and reverse transcriptionpolymerase chain reaction (PCR) Amplification (RT-PCR)

Genomic DNAs were isolated from fibroblasts, as described (Tanoue et al. 1990b). Total RNAs were isolated from fibroblasts using Isogen reagents (Nippon Gene, Tokyo, Japan) according to the manufacturer's instructions.

Synthesis of cDNA was carried out with random hexamers, and PCR was performed using specific primers (5' primers, F1, F2, F3, and F4; 3' primers, R1, R2, R3, and $\mathrm{R} 4$, shown in Table 1) for 30 cycles at $94^{\circ} \mathrm{C}$ for $1 \mathrm{~min}$, at $50^{\circ} \mathrm{C}$ and $55^{\circ} \mathrm{C}$ for $2 \mathrm{~min}$, and at $72^{\circ} \mathrm{C}$ for $5 \mathrm{~min}$. The PCR products were analyzed on agarose gels and subjected to sequencing directly or after subcloning into plasmids using a 377A DNA autosequencer (PE Biosystems, Tokyo, Japan).

Amplification and analysis of genomic DNA

Genomic DNA was amplified using two primers which correspond to the normal sequence of the human prolidase gene, as described (Tanoue et al. 1990a). The 5' primer IVS 7 corresponded to the sequence in intron 7 , and the $3^{\prime}$ primer IVS 8 to the sequence in intron 8 , as shown in Table 1 . The amplified DNA fragment was digested with the restriction enzyme DdeI and analyzed on a $2 \%$ agarose gel.

\section{Results and discussion}

Prolidase activity was measured in the fibroblasts from a patient with prolidase deficiency, using the method of Endo et al. (1990), as previously described. No prolidase activity was detectable in the fibroblasts, consistent with earlier re-

Table 1. Oligonucleotide primers used for polymerase chain reaction (PCR)

\begin{tabular}{|c|c|c|c|}
\hline & Primer sequence & Location & Fragment size \\
\hline \multicolumn{4}{|c|}{ Fragment 1} \\
\hline F1 & 5 ' TGACGCCGGTGCCGGGCGAA3' & $-21--2$ & \multirow{2}{*}{$420 \mathrm{bp}$} \\
\hline $\mathrm{R} 1$ & 5 ' GGCAATCTCATCTACGTACT3' & $380-399$ & \\
\hline \multicolumn{4}{|c|}{ Fragment 2} \\
\hline $\mathrm{F} 2$ & 5 'AAGATCCATTCCAAGGAGCA3' & $327-346$ & \multirow{2}{*}{$650 \mathrm{bp}$} \\
\hline $\mathrm{R} 2$ & 5 ' CACCAGTCACCTGGCTTCAT3' & $957-976$ & \\
\hline \multicolumn{4}{|c|}{ Fragment 3} \\
\hline F3 & 5 ' AGTGGTGAGAACTCAGCCGT3 ' & $738-757$ & \multirow{2}{*}{$391 \mathrm{bp}$} \\
\hline R3 & $5^{\prime}$ GCACGTCAATGCCCAGGAAG3 ' & $1110-1129$ & \\
\hline \multicolumn{4}{|c|}{ Fragment 4} \\
\hline F4 & 5 ' TGTTTATGCCTCACGGGCTM3' & $1085-1104$ & \multirow{2}{*}{425 bp } \\
\hline R4 & 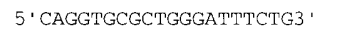 & $1490-1509$ & \\
\hline \multicolumn{4}{|l|}{ Exon 8} \\
\hline $5^{\prime}$ & 5 ' TCTGCAGTGCCTCCTGAAAG3 ' & IVS 7 & \multirow[t]{2}{*}{$220 \mathrm{bp}$} \\
\hline $3^{\prime}$ & 5 ' ATCCTGGAAACCCTTCATCC ${ }^{\prime}$ & IVS 8 & \\
\hline
\end{tabular}

Nucleotide positions in parentheses refer to cDNA position (Endo et al. 1990) and Genome sequence (Tanove et al. 1990a) sults in RBCs (Endo et al. 1990). The prolidase cDNA was amplified by RT-PCR in four fragments, a 420-bp fragment from nucleotides -21 to 399 , a 650-bp fragment from nucleotides 327 to 976 , a 391-bp fragment from nucleotides 738 to 1129, and a 425-bp fragment from nucleotides 1085 to 1509 . All fragments from the patient's fibroblasts were of the same size as those from the normal control fibroblasts.

Sequencing of the amplified cDNA from the patient revealed a single-base substitution (C-to-T at nucleotide position 551) that resulted in a stop codon from arginine at amino acid residue 184 (Fig. 1). This replacement was detected in all subclones after amplification from the patient's fibroblasts.

The deduced polypeptide consisted of 183 amino acid residues with a calculated molecular mass of approximately $20 \mathrm{kDa}$. This is different from the size of wild-type prolidase, which is 493 amino acid residues with a calculated molecular mass of $54 \mathrm{kDa}$.

To confirm that the substitution was not caused by PCR or cloning artifacts, and that it was homozygous, genomic DNA from the patient's fibroblasts was analyzed. Since the nucleotide substitution is present in exon 8 of the PEPD gene (Tanoue et al. 1990a), the nucleotide sequence includ-

\section{Normal}
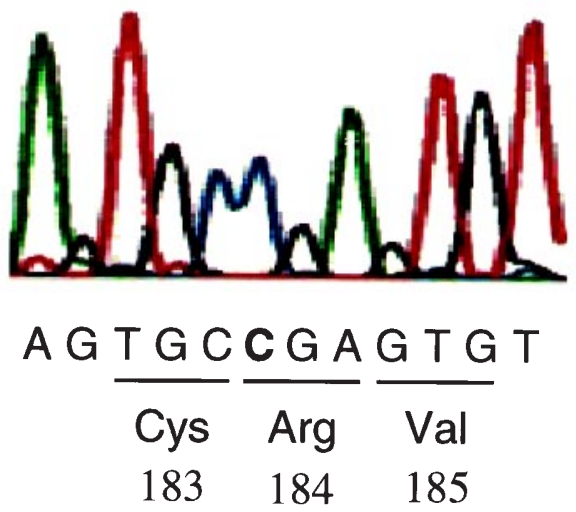

\section{Patient}

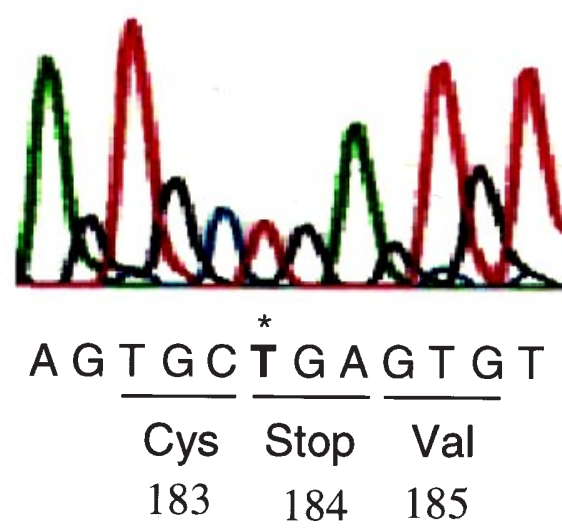

Fig. 1. Partial nucleotide sequences of the normal and mutated prolidase cDNA. The nucleotide sequence of the mutated region is shown. The sequence of mutated cDNA from the patient is compared with that of the control. The single base substitution of C-to-T at position 551 is indicated by an asterisk. The deduced amino acid sequence with the number of the amino acid residue is shown under the nucleotide sequence 
ing exon 8 was amplified with the primers. Using this primer set, we amplified a 220-bp fragment in the control and the patient. The C-to-T substitution at position 551 in exon 8 resulted in the creation of a $D d e I$ site, which cleaves at the 5'-CTGA-3' but not the 5'-CCGA-3' sequence. When amplified DNA from the patient was digested with DdeI, 160-bp and 60-bp fragments were generated (data not shown). On the other hand, the 220-bp fragment from the control remained unchanged after digestion with $D d e I$. This result indicates that this patient possesses a homozygous allele and that the substitution was not caused by a PCR artifact of the mRNA.

The novel PEPD mutant allele we have identified is a nonsense mutation at nucleotide position 551 in exon 8 of the PEPD gene. The substitution occurs as a consequence of a mutation involving the $\mathrm{CpG}$ dinucleotide, a position that is known to be a hot spot for point mutations because of spontaneous deamination of methylated cytosines (Coulondre et al. 1978). This mutation would cause the generation of a truncated polypeptide with a molecular mass of $20 \mathrm{kDa}$. Since we were not able to detect CRMs by Western blot analysis (Endo et al. 1990), it seems that this mutant protein may be very unstable in vivo.

Eight mutations have been identified in patients with prolidase deficiency to date: two deletions ( $\Delta$ exon 14 , $\Delta$ Glu452 or 453), mutations at two splicing sites (IVS6SA2/ IVS6SA2, IVS4SA1), and missense mutations (Asp276Asn, Gly448Arg, Arg184Gln, Gly278Asp) (Tanoue et al. 1990a,b, 1991; Ledoux et al. 1994, 1996). The patient with the nonsense mutation of C-to- $\mathrm{T}$ at nucleotide position 551 exhibited a severe phenotype, whereas the patient with the missense mutation at nucleotide position 551 (Arg184Gln) remained asymptomatic with residual prolidase activity (Ledoux et al. 1994, 1996). Mutations at position 551 are located in a non-conserved region of the protein, distal from the active site (Mock and Zhuang 1991). The nonsense mutation does affect the stability of the catalytically inactive enzyme, whereas the missense mutation at that position may not affect the stability.

Further analysis of more patients may be necessary to elucidate the relationship between the genotype and the phenotype, and it is hoped that the characterization of PEPD mutations may lead to predictive tests that distinguish severe from mild or asymptomatic cases. tance.

This work was supported in part by a Grant-in-Aid for Science Research from The Ministry of Education, Science, Sports and Culture of Japan, the Organization for Pharmaceutical Safety and Research (OPSR) of Japan, and the Organized Research Combination System from the Science and Technology Agency of Japan.

\section{References}

Coulondre C, Miller JH, Farabaugh PJ, Gilbert W (1978) Molecular basis of base substitution hotspots in Escherichia coli. Nature 274:775-780

Endo F, Tanoue A, Kitano A, Arata J, Danks DM, Lapiere CM, Sei Y, Wadman SK, Matsuda I (1990) Biochemical basis of prolidase deficiency. Polypeptide and RNA phenotypes and the relation to clinical phenotypes. J Clin Invest 85:162-169

Isemura M, Hanyu T, Gejyo F, Nakazawa R, Igarashi R, Matsuo S, Ikeda K, Sato Y (1979) Prolidase deficiency with imidodipeptiduria. A familial case with and without clinical symptoms. Clin Chim Acta 93:401-407

Isemura M, Hanyu T, Ono T, Igarashi R, Sato Y, Gejyo F, Nakazawa R, Miyakawa T, Takagi T, Kuboki Y, Sasaki S (1981) Studies on prolidase deficiency with a possible defect in collagen metabolism. Tohoku J Exp Med 134:21-28

Ledoux P, Scriver C, Hechtman P (1994) Four novel PEPD alleles causing prolidase deficiency. Am J Hum Genet 54:1014-1021

Ledoux P, Scriver CR, Hechtman P (1996) Expression and molecular analysis of mutations in prolidase deficiency. Am $\mathrm{J}$ Hum Genet 59:1035-1039

Mock WL, Zhuang H (1991) Chemical modification locates guanidinyl and carboxylate groups within the active site of prolidase. Biochem Biophys Res Commun 180:401-406

Ogata A, Tanaka S, Tomoda T, Murayama E, Endo F, Kikuchi I (1981) Autosomal recessive prolidase deficiency. Three patients with recalcitrant ulcers. Arch Dermatol 117:689-697

Oono T, Fujiwara Y, Yoshioka T, Arata J (1997) Prolidase activity in chronic wound and blister fluids. J Dermatol 24:626-629

Tanoue A, Endo F, Matsuda I (1990a) Structural organization of the gene for human prolidase (peptidase D) and demonstration of a partial gene deletion in a patient with prolidase deficiency. J Biol Chem 265:11306-11311

Tanoue A, Endo F, Kitano A, Matsuda I (1990b) A single nucleotide change in the prolidase gene in fibroblasts from two patients with polypeptide positive prolidase deficiency. Expression of the mutant enzyme in NIH 3T3 cells. J Clin Invest 86:351-355

Tanoue A, Endo F, Akaboshi I, Oono T, Arata J, Matsuda I (1991) Molecular defect in siblings with prolidase deficiency and absence or presence of clinical symptoms. A 0.8 -kb deletion with breakpoints at the short, direct repeat in the $P E P D$ gene and synthesis of abnormal messenger RNA and inactive polypeptide. J Clin Invest 87:11711176

Acknowledgements We are grateful to Miss Fujiki for technical assis- 